\title{
The ABCD of DALK
}

\author{
Jod Mehta
}

Corneal and External Eye Disease Department, Singapore National Eye Centre, Singapore

DOl: https://doi.org/10.17925/USOR.2017.10.02.93

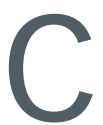

orneal transplantation has changed rapidly over the last 20 years. In spite of the fact that endothelial keratoplasty has become the most dominant procedure, deep anterior lamellar keratoplasty (DALK), the anterior lamellar procedure, has not become so popular. There are several reasons for this, and this editorial will attempt to give some useful tips, that may not be found in papers or books, in the hope of helping people performing or starting off on their DALK journey.

\section{Keywords}

Corneal transplantation, DALK, DALK technique, pearls for DALK

Disclosure: Jod Mehta has nothing to disclose in relation to this article. No funding was received for the publication of this article. This article is a short opinion piece and has not been submitted to external peer reviewers.

Authorship: All named authors meet the International Committee of Medical Journal Editors (ICMJE) criteria for authorship of this manuscript, take responsibility for the integrity of the work as a whole, and have given final approval to the version to be published.

Open Access: This article is published under the Creative Commons Attribution Noncommercial License, which permits any non-commercial use, distribution, adaptation and reproduction provided the original author(s) and source are given appropriate credit.

Received: August 2, 2017

Published Online: September 26, 2017 Citation: US Ophthalmic Review, 2017;10(2):93-4

Corresponding Author: Jod Mehta, Corneal and External Eye Disease Department, Singapore National Eye Centre, 11 Third Hospital Avenue, Singapore 168751. E: jodmehta@gmail.com
Over the last two decades, deep anterior lamellar keratoplasty (DALK) has re-emerged as a procedure of choice, for patients with corneal stromal disease, requiring corneal transplantation. Despite the fact that there are distinct advantages of DALK over penetrating keratoplasty (PK), the uptake of the procedure worldwide, has been very variable. The advantages of DALK include less intraocular complications (since it is predominantly an 'extra-ocular' procedure), fewer postoperative complications, the eye being tectonically stronger than PK, less risk of allograft rejection, better endothelial cell count postoperatively compared to PK, and less rigid requirements for the donor cornea. For DALK, I routinely use tissue that is 10-14 days old from harvest, with low endothelial cell count. This expands the donor pool, 'saving the better tissue' for endothelial keratoplasty.

Despite all the advantages of DALK over PK, the outcomes reported in the literature are very variable. The Singapore Corneal Transplant Database tracked outcomes of DALK over 16 years, and overwhelmingly shows better graft survival compared to PK. ${ }^{1}$ This has a profound effect on my surgical workload, where anterior lamellar keratoplasty accounts for $30 \%$ of all my transplant cases and is the second most common transplantation procedure I perform, behind Endothelial keraoplasty (58\%). PK being relegated, well and truly, to third place. However, data from the Australian Corneal Graft Registry shows worse outcomes of DALK compared to PK and in the US, DALK only accounts for $2 \%$ of all transplant procedures performed (EBAA 2015 Data). ${ }^{2}$

The vast differences in outcomes and also in the percentage of cases undergoing DALK in different centers is certainly down to the complexity of the procedure and the familiarity of how to handle complications associated with the procedure. I would like to say that there is a learning curve to the procedure, but I really do not think there is! A learning curve implies that once a person has gained sufficient experience and is on the plateau at the top, cases become straightforward. However, this appears not to be the case with DALK, and even experienced surgeons can get caught out. The learning curve however, is in handling complications when they occur or preventing the complications from happening. In this editorial, we will cover four tips, i.e., the 'ABCD', to help surgeons perform DALK. This is not an exhaustive list but just some tips I have found useful.

A: A is for anesthesia and attitude. It is important to have the appropriate mind-set before surgery. If you are regularly performing PK now, and want to start DALK, you have nothing to lose to try, since at worst, the patient will get a PK, which you were going to perform anyway. For every patient with stromal disease I routinely see in clinic who requires a corneal transplant, I will consider doing a DALK procedure. Everything is DALK-able! It is also important to have the correct anesthesia. It is not easy to perform a procedure on Descemet's membrane (DM) with the patient moving. Ideally do your cases under a general anesthesia with paralysis. It is important to ensure that there is no excessive pressure in the anterior chamber, since this will increase the risk of rupture of the DM. Give yourself time to perform the procedure. DALK will take longer than a routine PK.

$\mathrm{B}$ : $\mathrm{B}$ is for balance and be calm. It is important to have balance in the anterior chamber. The deeper you go with your dissection the more you will see the DM bulging. Ideally use a non-compressive 
Figure 1: Deep anterior lamellar keratoplasty technique

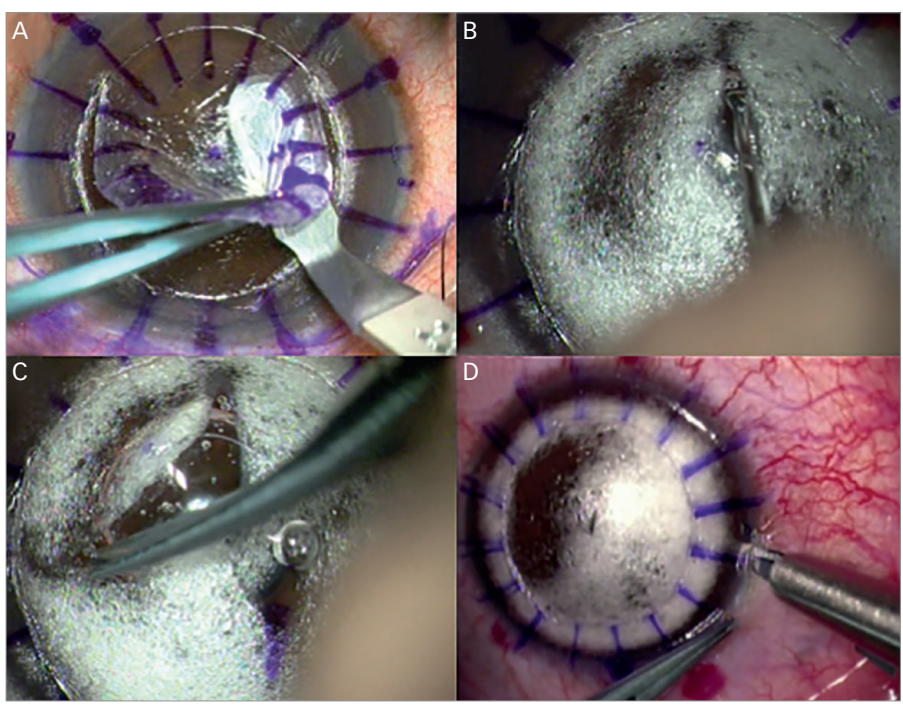

A: Removal of the anterior 30-50\% of corneal thickness consistently, allows access to the posterior 200 microns of the cornea. B: Use of Marginal Dissector (ASICO, Westmont, IL, US) to gently separate Descemet's membrane (DM) from the posterior stroma. C: Anwar scissor (Duckworth \& Kent, Baldock, UK) only allow movement of one blade, hence reducing risk of inadvertent DM rupture. D: Paracentesis after 'brave slash' reduces risk of DM rupture by decompressing the anterior chamber.

globe speculum. I routinely use a Jaffe speculum to avoid excessive pressure on the globe during surgery, which will raise the intraocular pressure (IOP). It is important to decompress the anterior chamber to reduce the IOP and prevent the bulge. This will ensure the DM is more lax, and reduce the risk of rupture. Often, during a case, I will decompress the anterior chamber a few times since it will reform with aqueous during surgery. If you perforate the DM, which will definitely happen sometime in your career, keep calm. Stop and assess the situation. The management of a perforation will depend on the time during the procedure that it has occurred, but in the majority of cases, the case can continue without conversion to a PK. In cases of micro-perforation, it is always best to use an interrupted technique when suturing, to help control the tension in the graft. If I perforate early in the procedure, I will often suture the graft with the anterior chamber full of air. This helps to tamponade the DM against the stroma, but the air must be removed at the end of the case.

C: C is for consistency. Try to make some of the procedure consistent regardless of which technique you will subsequently be using. I will always remove at least $30-60 \%$ of the cornea first, before tackling the posterior stroma. This is irrespective of whether I am performing a routine 'big bubble' DALK in a patient with keratoconus, or doing a DALK in a patient with previous hydrops, or doing a DALK in a patient with active infectious keratitis. By repeating the first steps of the procedure multiple times, I do not have to change my technique during more routine cases. I am also less tense in more complex cases. Tectonic cases with full thickness perforation are the exception to this statement, where I do alter my technique. For the vertical lamellar depth cuts, I use a Hanna Trephine System (Moria, Antony, France) since it has excellent accuracy on the vertical cut. However, you can use other guarded trephines or a femtosecond laser to achieve accurate
Figure 2: Alternative deep anterior lamellar keratoplasty techniques

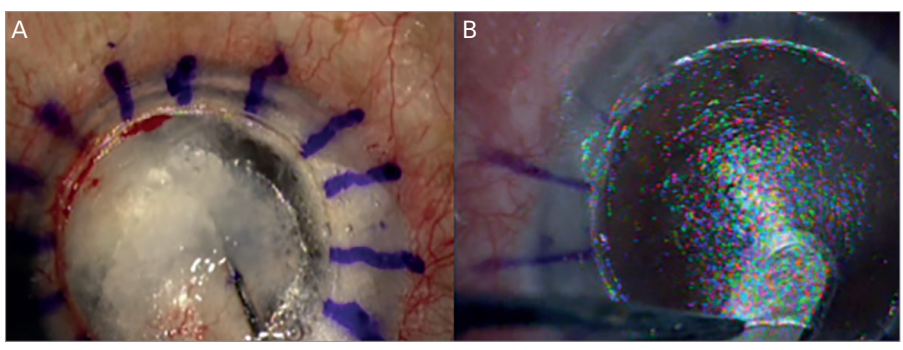

A: Viscobubble technique using a combination of air first followed by visco-elastic can allow dissection down to Descemet's membrane (DM) by opening up deeper planes. B: A good manual technique is important to learn as a 'fail safe'. It will allow you to get a smooth interface 50 microns from DM.

cuts as well. It is also important to be consistent with the instruments you use. I use the same instruments on every single case so that I am familiar with how they work and how they feel. I prefer to use very fine forceps to grip the stroma during dissection, normally $0.10 \mathrm{~mm}$ Bonn forceps (Duckworth \& Kent, Baldock, UK). They are atraumatic for the cornea and allow you to feel the tension on the stromal tissue. For the initial dissection, I will use a sharp $2.3 \mathrm{~mm}$ crescent blade made by BD (Chicago, US). For the stromaectomy around the graft edge I use the Anwar scissors (Duckworth \& Kent, Baldock, UK). For my deep lamellar dissection and DM bearing surgery, I use a marginal dissector (ASICO, Westmont, IL, US). If I am performing 'big bubble' or 'viscobubble' I will use the Tan DALK Cannula (ASICO). You can off course perfrom DALK using other instruments, but I have found these the most useful in reducing intra-operative complications.

D: D is for Descemet's membrane. A lot of people think, if they do not 'bear' the DM during the procedure, they have failed. This is not true. A good deep manual technique will also give good visual acuity. However, it may take longer to achieve that good vision. There are several techniques reported in the literature to bear the DM. These include the 'big bubble' technique using forceful injection of air, visco-elastic dissection, a combination of visco-elastic and air, manual layer-by-layer dissection, and the use of balanced salt solution to hydrate the cornea. I will use a combination of these techniques to achieve DM bearing DALK. It is important to be well versed or at least familiar with all these techniques. However, in other cases I will aim to perform a good deep manual technique - using our previously published technique, aiming to be approximately 50 microns from the DM. The decision on which technique to perform should be made in the clinic not in the operating theatre. Assessing the depth of posterior scars from multiple angles and the depth of lesions using a slit beam or anterior segment optical coherence tomography (OCT), can be helpful. Indecision at the time of surgery about what kind of DALK you are trying to achieve, will lead to indecision during the surgery. If you can do a good manual DALK, this will always be your back up plan.

The future of DALK has to be to de-skill the surgery further. Technology may play a significant role, such as the use of femtosecond lasers or integrated OCT. This will then hopefully increase the widespread dissemination of this technique. $\square$ 